\title{
Development of a statistical reduction method for the Palomar Fiber Nuller
}

\author{
Charles Hanot $^{a}$, Bertrand Mennesson ${ }^{b}$, Eugene Serabyn ${ }^{b}$, Stefan Martin $^{b}$, Kurt Liewer ${ }^{b}$, Frank \\ Loya $^{b}$, Dimitri Mawet ${ }^{b}$, Pierre Riaud $^{a}$, Olivier Absil ${ }^{a}$ \\ ${ }^{a}$ Institut d'Astrophysique et de Géophysique, University of Liège, 17 allée du 6 Août, B-4000, \\ Sart Tilman, Belgium; \\ ${ }^{b}$ Jet Propulsion Laboratory, California Institute of Technology, 4800 Oak Grove Drive, \\ Pasadena, California 91109, USA
}

\begin{abstract}
A unique statistical data analysis method has been developed for reducing nulling interferometry data. The idea is to make use of the statistical distributions of the fluctuating null depths and beam intensities to retrieve the astrophysical null depth in the presence of fluctuations. The approach yields an accuracy much better than is possible with standard data reduction methods, because the accuracy of the null depth is not limited by the sizes of the phase and intensity errors but by the uncertainties on their statistical distributions. The result is an improvement in the instrumental null depth measurement limit of roughly an order of magnitude. We show in this paper that broadband null depths of $10^{-4}$ can be measured in the lab with our infrared Fiber Nuller without achromatic phase shifters. On sky results are also dramatically improved, with measured contrasts up to a couple of $10^{-4}$ with our instrument mounted on the Hale telescope at the Palomar Observatory. This statistical analysis is not specific to our instrument and may be applicable to other interferometers.
\end{abstract}

Keywords: Statistics, Nulling interferometry, Coronagraphy, stellar diameters

\section{INTRODUCTION}

Since the first discovery of an exoplanet around a solar-type star in $1995,{ }^{1}$ the quest to find earth-like exoplanets and more importantly detect the presence of life on them became a major topic in astophysics. However, direct imaging of such systems is very challenging because of the high spatial resolution and dynamic range required. One possible way to overcome these difficulties is to use nulling interferometry. ${ }^{2}$ In this approach, one combines destructively the light coming from two or more apertures in order to dim the bright on-axis target and reveal faint objects or structures in its immediate vicinity.

Analysing interferometric data in general, and nulling interferometric data in particular, ${ }^{3}$ is a complex task because accurate calibration of the instrument is needed to extract the scientific information. In the case of interferometric nulling, the quantity of interest is the astrophysical null depth (ND), which directly relates to the target's spatial brightness distribution. In practice however, the measured interferometric null depth (the inverse of the stellar light rejection ratio) is not strictly equal to the astrophysical ND. It also incorporates the effect of instrumental noise and bias sources such as phase errors, intensity mismatch and global intensity fluctuations. Until now it was considered that for proper determination of the astrophysical ND, the mean values of these instrumental error sources needed to be accurately known. ${ }^{4,5}$ The classical method used for deriving astrophysical ND - and visibilities - is then to average different sequences recorded on the science star and estimate the instrumental bias by observing a calibrator star. This technique has been extensively used for years for both classical and nulling interferometry, but suffers from well known limitations: (i) the accuracy is limited by the stability of the observing conditions, (ii) the final accuracy depends on the scientific knowledge of the calibrator star, (iii) calibrators observations are time consuming. What we propose here is to use the interferometer null depth distribution in order to increase the sensitivity and stability of astrophysical null measurements. The principle is to record the null depth fast temporal fluctuations and retrieve the underlying astrophysical information

Further author information: (Send correspondence to Charles Hanot) Charles Hanot: E-mail: hanot@astro.ulg.ac.be, Telephone: +32 (0)4 3669768

Optical and Infrared Interferometry II, edited by William C. Danchi, Françoise Delplancke, Jayadev K. Rajagopal, Proc. of SPIE Vol. 7734, 77342S · C 2010 SPIE · CCC code: 0277-786X/10/\$18 · doi: 10.1117/12.857014 
by modeling the histogram of observed null values. Using this statistical analysis, we show that it is possible to retrieve the astrophysical ND with a much better accuracy than with classical approaches. Moreover, initial stellar observations indicate that such a method does not require any calibration down to null depth measurement accuracies as low as a couple $10^{-4}$. The goal of this paper is twofold. We first explain the principle and theory of our interferometric data analysis strategy based on statistics. We then apply these results to astronomical data obtained with the Palomar Fiber Nuller (PFN) ${ }^{6-8}$ a transportable nulling coronagraph developed at the Jet Propulsion Laboratory. It must be emphasized that the reduction method we have developed can be applied to null / visibility measurements in general, as long as some fringe tracking capability and single-mode filtering of the wavefronts are available. For example, Riaud and Hanot in a forthcoming paper, use our technique applied to the measurement of Cepheid stellar diameters with Long Baseline Inerferometry. ${ }^{9}$

\section{NULLING STATISTICS}

\subsection{Null Expression}

We establish here the expression of the observed null depth as a function of the major error sources found in an interferometer. In the case of two planar monochromatic wavefronts recombined into a single-mode waveguide, the output intensity measured at time $t$ is given by: ${ }^{4}$

$$
\begin{aligned}
I_{ \pm}(t) & =\frac{1}{2}\left[I_{1}(t)+I_{2}(t) \pm 2 \cos (\Delta \phi(t)) \cos \left(\alpha_{r o t}\right) \sqrt{I_{1}(t) I_{2}(t)}\right] \\
& =I(t)\left[1 \pm \cos (\Delta \phi(t)) \cos \left(\alpha_{r o t}\right) \sqrt{1-\left(\frac{\delta I(t)^{2}}{4}\right)}\right]
\end{aligned}
$$

where $I_{1}(t)$ and $I_{2}(t)$ are respectively the individual intensities of beam 1 and $2, I(t)=\left(I_{1}(t)+I_{2}(t)\right) / 2$ is the averaged intensity, $\delta I(t)=\left(I_{1}(t)-I_{2}(t)\right) /\left(I_{1}(t)+I_{2}(t)\right)$ is the relative intensity mismatch, $\Delta \phi(t)=\phi_{1}(t)-\phi_{2}(t)$ their relative phase delay and $\alpha_{\text {rot }}$ their relative polarization rotation angle.

The null depth, defined as the inverse of the rejection ratio, is given by:

$$
N(t)=\frac{I_{-}(t)}{I_{+}(t)}
$$

If $\Delta \phi(t), \delta I(t)$ and $\alpha_{\text {rot }}$ are all small, the null depth can be approximated by:

$$
N(t) \simeq\left(\frac{\delta I(t)^{2}+\Delta \phi(t)^{2}+\alpha_{\text {rot }}(t)^{2}}{4}\right)
$$

Eq. 4 is valid for point sources, and can be used for small null depth values, typically $<0.01$.

If the source has a finite extent, the observed null depth now also depends on the astrophysical null $N_{a}$, purely set by the brightness distribution of the target *. Using Eq. 4, it has been shown that for small values of $N_{a}$, the observed null depth can be expressed as: ${ }^{4}$

$$
N(t) \simeq\left[\frac{\delta I(t)^{2}}{4}+\frac{\Delta \phi(t)^{2}}{4}+\frac{\alpha_{r o t}(t)^{2}}{4}+N_{a}\right]
$$

\footnotetext{
${ }^{*}$ The astrophysical ND $N_{a}$ can be expressed in terms of the complex visibility $\mathrm{V}$ of the target at the observing baseline: $N_{a}=(1-|V|) /(1+|V|)$
} 
In some interferometers, the bright and destructive outputs are not measured simultaneously. In that case, one actually measures a null value:

$$
\begin{aligned}
N(t) & =\frac{I_{-}(t)}{I_{+}(t+\Delta t)} \\
& \simeq I_{N}(t, \Delta t)\left[\frac{\delta I(t)^{2}}{4}+\frac{\Delta \phi(t)^{2}}{4}+\frac{\alpha_{r o t}^{2}}{4}+N_{a}\right]
\end{aligned}
$$

where

$$
I_{N}(t, \Delta t)=\frac{I(t)}{I(t+\Delta t)}
$$

is the normalized intensity uncertainty.

The last term influencing the null depth measurement - especially for faint stars having low signal-to-noise ratios (SNR) - comes from the instrumental background. Denoting $N_{B}$ the background induced null depth uncertainty:

$$
N_{B}(t)=\frac{I_{B}(t)-\left\langle I_{B}(t)\right\rangle}{I_{+}(t+\Delta t)}
$$

with $\left\langle I_{B}(t)\right\rangle$ the estimated background intensity at time $t$ and $I_{B}(t)$ its actual value, the measured null $N(t)$ is finally given by the expression:

$$
N(t) \simeq I_{N}(t, \Delta t)\left[\frac{\delta I(t)^{2}}{4}+\frac{\Delta \phi(t)^{2}}{4}+\frac{\alpha_{r o t}^{2}}{4}+N_{a}\right]+N_{B}(t)
$$

From now on, we refer to the expression in Eq. 9 as the "nulling function".

\subsection{Nulling distributions}

Until today, it was common to consider that the astrophysical null depths $N_{a}$ were found by averaging the null depth measurements $N(t)$ over a certain period of time. However, doing so, the astrophysical sensitivities of the nulling interferometers are limited by the mean phase, intensity and background errors. It is however possible to use the fluctuating noises in order to get the astrophysical information. For that, we need to use the statistical distribution of the null depth measurement instead of the mean values. We show in the next section how to use such distributions to extract the astrophysical signal out of the noise fast fluctuations.

\subsubsection{Statistical Self Calibration Method}

Equation 9 defines the dependence of the fluctuating null depth over the time as a function of the different noise sources and the astrophysical signal. If we had a perfect system free of any noise sources, the Null depth distribution would be a simple dirac function centered on the astrophysical null. Because of the noises fast fluctuations, this null depth histogram has a much more complex shape. If we decompose the problem into the different noise contributors and that we find their distributions, we will also find the corresponding astrophysical null depth despite the fluctuations of the measured null depth. The principle of our method is to generate random vectors for each contributors of Eq. 9 that have same length as the dataset and, after combining them, compare the resulting null depth distribution with the observed one. One could think that the number of free parameters defining the null depth histogram is too large to make this method usable but most of the contributors appearing in Eq. 9 are either monitored or can be neglected. Indeed, for most interferometers, the individual intensities of the beams and the background level are monitored. $I_{N}(t, \Delta t), \delta I(t)$ and $N_{B}(t)$ distributions are therefore known and can be directly injected into the nulling function. The rotation of the polarization on the other hand can be considered as constant during an observing run. For some interferometric designs using off-axis single mode fiber beam recombination, ${ }^{7}$ this term is even negligible compared to other noise sources. Therefore, it can be either calibrated before the observations or is negligible but in all cases, it can be ignored in the nulling distribution. 




Figure 1. Fit using the statistical self calibration method on a dataset obtained on $\alpha$ Boo with the PFN in July 2009. The astrophysical ND corresponding to the best fit is $0.0132 \pm 0.0004$.

The only remaining distributions that need to be found are the phase error and the astrophysical nulling leakage. Some studies show that the residual phase error after an AO system or a fringe tracker follow normal distributions. ${ }^{10}$ The shape of the null depth distribution therefore only depends on three parameters that are the mean and the standard deviation of the phase error and the astrophysical null depth. To summarize, the statistical reduction method we hereby present simply consist in feeding Eq. 9 with the monitored $I_{N}(t, \Delta t), \delta I(t)$ and $N_{B}(t)$ distributions. Then to generate a random phase error vector following a normal distribution and having the same length as the dataset. And finally, to add the astrophysical null depth to the modeled distribution. We then try to find the set of free parameters ( the mean phase error $\mu_{\phi}$, the rms of the phase error $\sigma_{\phi}$ and $N_{a}$ ) that generate a null depth histogram that best fit the observed distribution.

In this approach, we do not make any assumptions on the distributions of the intensity mismatch, background and total intensity terms, which are assumed to be measured within the null sequences or close in time. We use the distributions actually observed for each of these quantities. In the case of the PFN for instance, interleaved $(<100 \mathrm{~ms})$ measurements of the individual beams, interferometric (close to null) and background intensities are recorded over sequences of a few minutes. Although the background and individual beam signals are not recorded exactly at the same time as the null, their distributions can be measured with very high fidelity. In order to analyze a sequence of observed null values, we use these observed distributions to generate a random null (Eq. 9) vector of the same size. We make two assumptions: (i) the differential phase follows a gaussian distribution, (ii) the individual beam intensities are uncorrelated. Besides its simplicity and computation speed, the main advantage of the self calibration statistical technique is that the data monitored by the instrument (i.e. the individual beam intensities and the background) are directly injected into the model. Therefore, no matter what the real distributions are for those terms, no bias is introduced into the modeled histogram. However, as the random generation of finite vectors produces slightly different histograms and best fit parameters for different seeds, this method adds some intrinsic uncertainty. This "fitting noise" - computed by generating many random phase vectors with the same distribution - adds quadratically to the statistical error appearing in any astrophysical observations. The final error bar on the astrophysical null is computed conservatively as the quadratic sum of the "external" error (scatter between $i$ individual null depth measurements, denoted $N_{a, i}$ ) and 
the "statistical" error defined as:

$$
\frac{1}{\sigma_{\text {stat }}^{2}}=\sum_{i=1}^{n_{o}} \frac{1}{\sigma_{\text {stat }, \mathrm{i}}^{2}}
$$

Figure 1 shows an example of histogram fitting obtained on $\alpha$ Boo with the Palomar Fiber Nuller (PFN) using the statistical self-calibration method.

These results illustrate a general characteristic of the histogram fitting approach: even though the mean measured null depth over the whole sequence is of the order of 0.1 , reliable measurement of astrophysical ND is possible at a much lower level (10 to 100), and with far better accuracy (see section 3.2).

\section{HYPOTHESIS VERIFICATION AND FIRST RESULTS WITH THE PFN}

In order to investigate the validity and actual accuracy our statistical data reduction based on null distribution fitting, we apply it to early astronomical data obtained with the PFN during a July 2009 observing run. Nine very bright stars have presently been observed with the PFN, and eight of them were clearly resolved. A complete summary and interpretation of the stellar nulls measured to date with the PFN will be presented in a forthcoming paper (Mennesson et al. in prep). In all cases, the stellar diameters derived from the measured astrophysical nulls are in excellent agreement with values previously reported by infrared long baseline interferometry, making us confident that the approach is sound. We present in this section some typical examples of the astrophysical ND and accuracy achieved so far when applying the statistical data reduction technique to PFN data. We then briefly discuss the current limitations and potential improvements.

\subsection{Hypothesis verification}

We explore in this section some possible limitations of the statistical data reduction technique, which may show up when trying to reliably measure astrophysical ND typically ten times deeper than presently done, i.e at the 0.0001 level and with comparable accuracy. Limitations arise from well identified sources: chromatic effects and deviation from the assumptions used in the modeling. There are only two assumptions made in the statistical self calibration technique: no temporal correlation between the individual beam intensities, and gaussian distribution of the differential phase. In the following, we investigate these different effects, assess their contributions to the final ND estimates, and suggest some mitigation techniques.

\subsubsection{Correlation issues}

In the statistical method (section 2.2.1), we made the assumption that the individual beam intensities were temporally uncorrelated, so we could compute the theoretical null distribution from the individual noise distributions without computing cross-correlated terms. On interferometers using single-mode fiber injections the intensity fluctuations are driven by the tip-tilt introduced by the atmospheric turbulence cells above the apertures. The optical/ near infrared coherence length of the atmosphere is generally much smaller than the distance between an interferometer apertures. Consequently, the correlation between the interferometric beam intensities is expected to be low. Even with the compact PFN system, the typical value for the Fried's radius $\left(70 \mathrm{~cm}^{11}\right.$ at 2.2 microns), is much smaller than the interferometric baseline of $3.4 \mathrm{~m}$. This suggest that the absence of correlation between the individual intensities is to first order justified both for the PFN, and long baseline interferometry in general.

Following the above argument that the beam intensity is primarily driven by the phases over the individual sub-apertures, no intensity correlation is expected for beams separated by more than a turbulence coherence length. However one can not eliminate possible common sources of intensity fluctuations over the different apertures, such as high altitude cirrus for instance.

The question of the correlation between the two beam's intensities would be trivial if we were able to monitor the two intensities at the same time, as done for some interferometers. ${ }^{12-14}$ Unfortunately this is not the case with the PFN and we need to find a way to assess this correlation by an indirect indicator. What we propose 


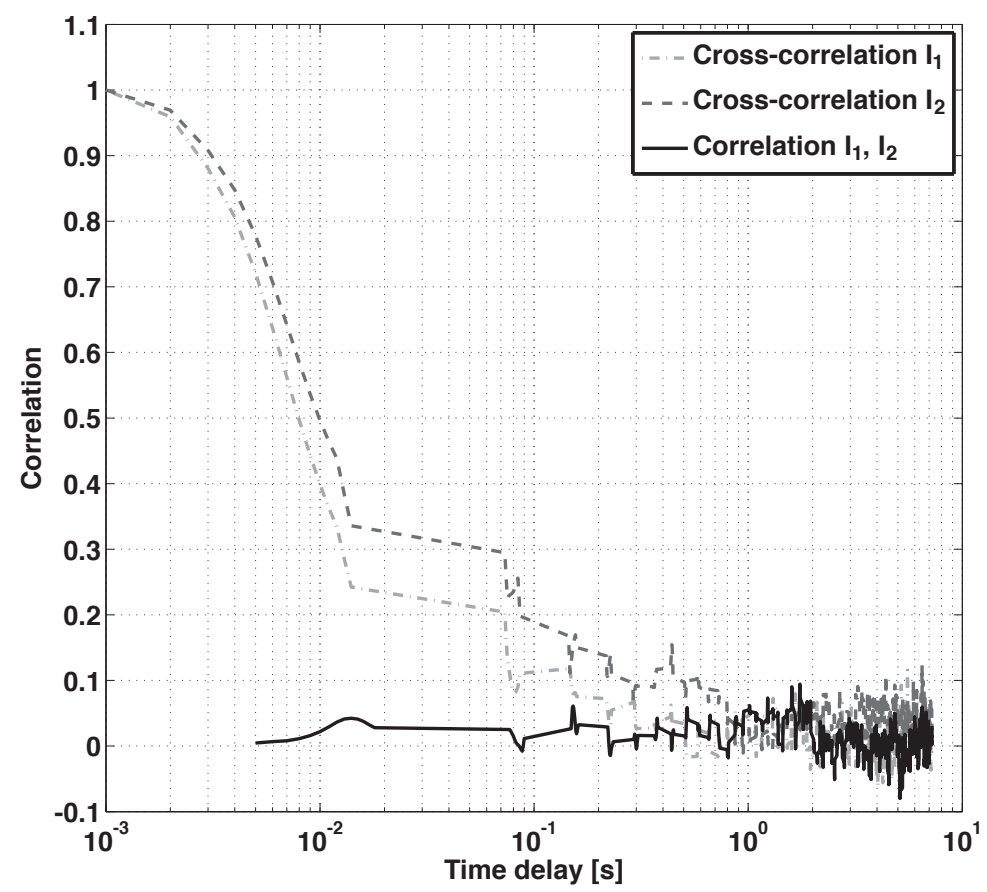

Figure 2. Graph comparing the auto-correlation of $I_{1}(t), I_{2}(t)$, and the cross-correlation of $I_{1}(t)$ and $I_{2}(t+\Delta t)$.

is to measure the correlation between $I_{1}(t)$ and $I_{2}(t+\Delta t)$ with the shortest possible time delay $\Delta t$ available in our observations (typically $5 \mathrm{~ms}$ ). We can then compare this cross-correlation with the value obtained for the autocorrelation of $I_{1}(t)$ (and $I_{2}(t)$ ) at similar time delays. We apply this method on datasets obtained with the PFN under excellent seeing conditions: seeing of $0.5 "$ and wind speed $\sim 10 \mathrm{~m} / \mathrm{s}$.

Figure 2 displays the auto-correlation of $I_{1}(t)$ (in light grey) and $I_{2}(t)$ (in dark grey) for time delays varying between $5 \mathrm{~ms}$ and about $7 \mathrm{~s}$. The autocorrelation decreases like a gaussian function down to $25 \%$ for $\Delta t=20 \mathrm{~ms}$ and below $10 \%$ for $\Delta t>100 \mathrm{~ms}$. The full width at half maximum (FWHM) is $\sim 10 \mathrm{~ms}$.

The black curve of Fig. 2 on the other hand, shows the cross-correlation of $I_{1}(t)$ with $I_{2}(t+\Delta t)$ with $\Delta t$ varying between $5 \mathrm{~ms}$ and $7 \mathrm{~s}$. It can be seen that for these delays, there is no correlation left between the two intensities while the autocorrelation of $I_{1}(t)$ (and $I_{2}(t)$ ) is significant for short time delays. This indicates a low correlation between $I_{1}(t)$ and $I_{2}(t)$.

Finally, in order to estimate the effect of intensity correlation on real stellar observations, we processed PFN data with the self-calibrated method using the two extreme cases of zero and perfect correlation. In all cases, the impact on the derived astrophysical ND was found below 0.001 .

\subsubsection{Differential phase distribution}

For the statistical reduction techniques presented, the differential phase - computed at the central observing wavelength, is assumed to exhibit a gaussian distribution over the nulling sequence recorded. The validity of this assumption is difficult to assess. As long as the instrument tracks around a constant optical path difference (OPD) position, it seems a reasonable assumption. In the case of the PFN, the two beams come from the same AO corrected wavefront. Tracking a single OPD comes down to the fact that the AO system, which acts as a fringe tracker, tries to maintain the same reference flat wavefront over the sequence, which is guaranteed by design. Some studies have shown that indeed, the phase residual after a AO system are gaussian which confirms our assumption. ${ }^{10}$ If for some reason the fringe tracker or AO system loses lock, or if the OPD is obviously oscillating between several distinct positions, the resulting distribution will no longer be gaussian, and the corresponding data should be discarded. The reduced Pearson $\chi^{2}$ defining the quality of the histogram fit (Equation 17) is a good quantitative tool to assess the validity of the gaussian OPD distribution. If measured $\chi^{2}$ 
are much larger than one, error bars on the final astrophysical ND should be increased accordingly. Determining the potential bias caused by any departure from a gaussian OPD distribution is beyond the scope of this paper, but will likely play a role for measuring reliable nulls at the $<0.0001$ level.

\subsubsection{Chromaticity effects}

Similarly, interferometric / nulling observations are conducted over a finite spectral bandwidth. We concentrate here on the effects of the chromatic phase term, expected to dominate over the chromatic aspects of intensity or polarization mismatch. For a polychromatic observation, the phase error $(\Delta \phi(t))$ is the sum of the piston error calculated at band center $\left(\Delta \phi_{c}(t)\right)$ and the chromatic phase error $\left(\Delta \phi_{\lambda}(\lambda, t)\right)$. The effect of differential phase on the null (Eq. 9) must now be integrated over the passband, and is given by:

$$
N_{\phi}(t)=\frac{\int_{\lambda_{\min }}^{\lambda_{\max }}\left(\Delta \phi_{c}(t)+\Delta \phi_{\lambda}(\lambda)\right)^{2} \eta(\lambda) \mathrm{d} \lambda}{4 \int_{\lambda_{\min }}^{\lambda_{\max }} \eta(\lambda) \mathrm{d} \lambda}
$$

where $\eta(\lambda)$ is the spectral transmission of the instrument. In order to simplify the development here after, we consider $\eta(\lambda)$ as constant over the bandpass. The upcoming discussion however remains valid for any $\eta(\lambda)$ distribution. Eq. 11 can be written as follows:

$$
N_{\phi}(t)=\frac{\Delta \phi_{c}^{2}(t)}{4}+\frac{\Delta \phi_{c}(t)}{2} \int_{\lambda_{\min }}^{\lambda_{\max }} \Delta \phi_{\lambda}(\lambda) d \lambda+\int_{\lambda_{\min }}^{\lambda_{\max }} \frac{\Delta \phi_{\lambda}^{2}(\lambda)}{4} d \lambda
$$
0 , and

Without any loss of generality, it is always possible to define the central wavelength so that $\int_{\lambda_{\min }}^{\lambda_{\max }} \Delta \phi_{\lambda}(\lambda) d \lambda=$

$$
N_{\phi}(t)=\frac{\Delta \phi_{c}^{2}(t)}{4}+N_{\text {chrom }}
$$

Where $N_{\text {chrom }}=\int_{\lambda_{\min }}^{\lambda_{\max }} \frac{\Delta \phi_{\lambda}^{2}(\lambda, t)}{4} d \lambda$ is the chromatic null bias . So even in the case where the differential phase at the center of the band is zero, a positive bias is present (either constant or slowly varying, see below), and one measures $N_{\phi}(t)=N_{\text {chrom }}$. This additive bias directly impacts the astrophysical ND measurement.

In the case of the PFN, this chromatic term is minimized by inserting glass pieces of different thickness in each of the two beams. The chromatic bias is experimentally found to be lower than 0.0001 in the laboratory (see Sect. 3.3) and will be the subject of an future paper (Hanot et al., in prep.). On the sky, the dispersive phase is no longer a static term coming from the instrument. It is also impacted by differential atmospheric refraction across the band, and varies over the night according to the target's position with respect to zenith. For instance, looking at a source 20 degrees of zenith, and observing with the PFN baseline parallel to the refraction direction (worse case), the atmospheric refraction over the full $\mathrm{K}$ band contributes an additional bias of 0.0009. Solutions exist to strongly reduce or completely eliminate this effect: disperse over several spectral bins, always orientate the interferometric baseline perpendicular to the refraction direction (trivial on a single-dish multi sub-aperture interferometer), or use atmospheric dispersion compensators at the telescope level. Finally, this refraction effect is fortunately very repeatable, and could be precisely calibrated by observing reference stars at the same zenith angle.

\subsection{Classical vs. Statistical reduction methods}

We use here a common set of five independent null sequences recorded on $\alpha$ Her in July 2009 with the PFN in July 2009, and compare the astrophysical NDs and accuracies derived from (i) the "classical" null (or visibility) data reduction method and (ii) from the histogram analysis method. 


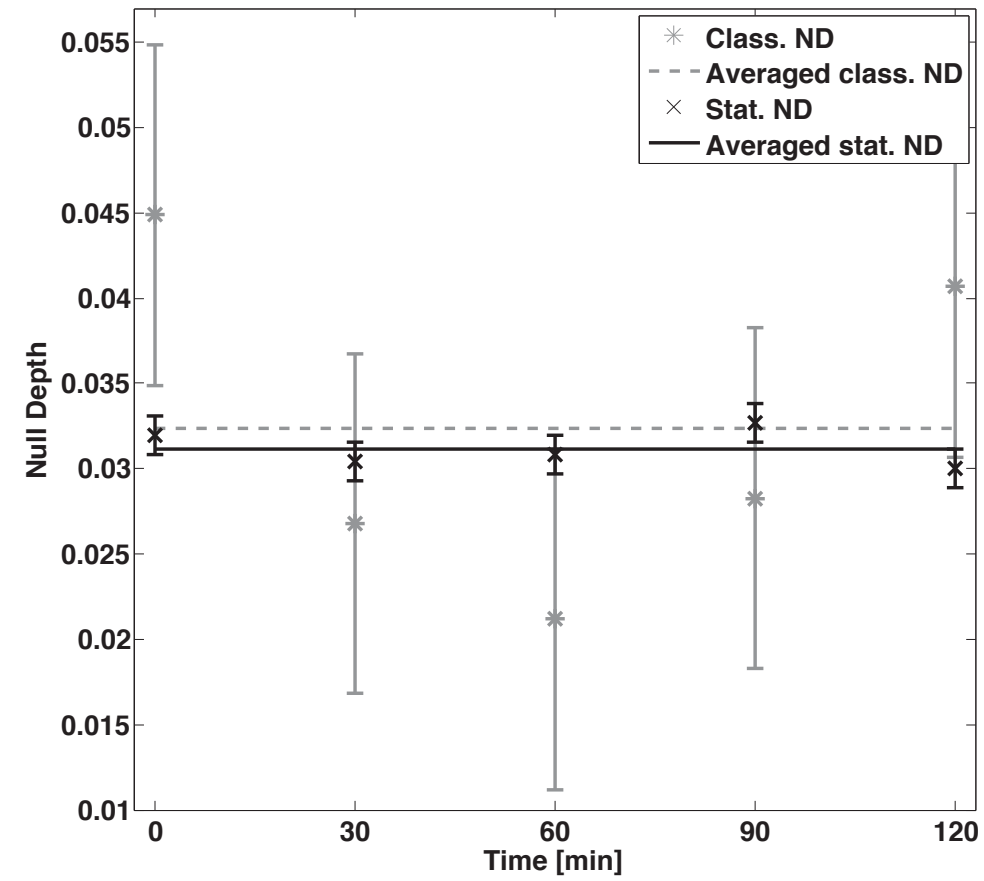

Figure 3. Comparison between astrophysical ND values obtained with both classical and statistical data analysis approaches on $\alpha$ Her.

\subsubsection{Classical reduction method}

Very few nulling data from ground based telescopes have been analysed so far, as only two nulling interferometers are operating: the Keck Interferometer Nuller ${ }^{3}$ and the MMT Nuller. ${ }^{15}$ Until today, the method used to analyse null data was analogous to that used for calibrating visibility measurements. The principle is to first evaluate the null depth observed on the science target by averaging the fluctuating instantaneous null depth over a significant number of points. This measurement is biased due to the fast fluctuations of phase and intensity errors. The same measurement is then conducted on a calibrator star of well known diameter, located close to the science target and with a similar magnitude at the wavelength of observation. For both stars, the measured null depth $\langle N(t)\rangle$ is the sum of the astrophysical null $N_{a}$ and the mean instrumental null $\left\langle N_{\text {Ins }}(t)\right\rangle$ over the sequence:

$$
\begin{aligned}
\langle N(t)\rangle & =N_{a}+\left\langle N_{\text {Ins }}(t)\right\rangle \\
\left\langle N_{\text {cal }}(t+\Delta t)\right\rangle & =N_{a, \text { cal }}+\left\langle N_{\text {Ins }, \text { cal }}(t+\Delta t)\right\rangle
\end{aligned}
$$

where the astrophysical ND $N_{a, c a l}$ on the calibrator star is supposed to be known a priori from its physical characterisitics or from independent interferometric observations. Therefore, assuming that the instrumental null is constant, one estimates the scientific target's astrophysical ND as:

$$
N_{a}=N_{a, c a l}+\langle N(t)\rangle-\left\langle N_{c a l}(t+\Delta t)\right\rangle
$$

Obviously, the accuracy on $N_{a}$ depends both on the calibrator's null uncertainty and on the stability of the instrumental null (or the ability to extrapolate its value accurately based on bracketing calibrator observations).

The method used to emulate a "classical analysis" of the nulling data is the following. We first reject the "bad" (large instantaneous nulls) data points within each dataset, both for the target and the calibrator. We only keep the data having null values comprised between the minimum measured null $N_{\min }$ and $N_{\min }+\sigma_{N}$, where $\sigma_{N}$ is the rms of the nulling data. The null depth of an individual object sequence is then computed as the mean of the remaining data points. The same approach is applied to both the scientific target and the calibrator data and the calibrated astrophysical ND is then computed using equation 16 . The grey stars in figure 
Table 1. Comparison between LD diameter found by long baseline interferometry, by the classical reduction and by the statistical data reduction method (using either the self calibration or the analytical fitting technique).

\begin{tabular}{llc}
\hline \hline Method & Name & ND [\%] \\
\hline LBI & $\alpha$ Her & $0.0325 \pm 0.0015$ \\
Classic & $\alpha$ Her & $0.0324 \pm 0.002$ \\
Stat. & $\alpha$ Her & $0.0312 \pm 0.00022$ \\
\hline
\end{tabular}

3 represent the calibrated null depth obtained with the classical data strategy on five consecutive $\alpha$ Her datasets. It illustrates that the classical method is very sensitive to the atmospherical conditions. Indeed, the null depth varies a lot within a relatively short timescale. Therefore, the precision on the astrophysical ND measurement for each individual sequence is poor with errorbars of $\pm 4.5 \times 10^{-3}$. Averaging over the 5 data points, we measure on $\alpha$ Her a leakage of $0.0324 \pm 0.002$. It is worth mentioning, that such low uncertainty on the null is equivalent to a 0.004 accuracy on the interferometric visibility. In comparison, the best $1 \sigma$ accuracy reported from long baseline interferometry is $\simeq 0.005,{ }^{16}$ and 0.002 for interferometric nulling of bright stars in the mid-infrared. ${ }^{3}$

\subsubsection{Statistical reduction method}

On the other side, the statistical data analysis uses the whole range of nulling values recorded and does not use nor require any calibration star. Using the same five $\alpha$ Her datasets, the individual astrophysical NDs measured using statistics have much smaller individual error bars, and are very stable over the whole two hours of observations (see Fig. 3). Taking the average of all five datasets, and computing the final error bar as described in section 2.3.1, one gets for $\alpha$ Her an estimated leakage $N_{a}=0.0312 \pm 0.00022$. The result we find is perfectly within the error-bars of the results found with long baseline interferometry (see Tab. 1) ${ }^{17}$ and the uncertainties on the measurement is 10 times smaller than what is found with the classical approach. Just to emphasize on this aspect once again, the corresponding error-bar in terms of visibilities is 0.00044 which is also 10 times better than any of the LBI results.

\subsubsection{Measuring deep nulls with high accuracy}

Figure 4 shows a series of null depth measurements carried on Vega with the PFN in July 2009. The apparent diameter of Vega is much smaller than that of $\alpha$ Her, providing a better test of the statistical method ability to measure deep nulls. The astrophysical ND predicted for PFN observations of Vega's photosphere alone, ${ }^{18}$ i.e. assuming no circumstellar emission, is 0.0005 . In comparison, the astrophysical ND derived from the PFN observations has a mean value of 0.0011 and is quite stable, with a standard deviation of only 0.0005 over the two hour sequence. The scientific significance of this paper will be discussed in a separate paper (Mennesson et al. in prep). It is worth noting that this long sequence comprises observations obtained with three different interferometric baseline orientations (30 degrees from each other), which is not the ideal way to assess the instrument ultimate stability performance at a given setting. However, it provides a conservative estimate of its accuracy since baseline rotation may cause additional null variations due to instrumental and/ or real astrophysical effects. The observed astrophysical ND fluctuations are also compatible with the quoted individual error bars, making us confident in the validity of the analysis. These initial measurements, together with those of another few stars (Mennesson et al. in prep) confirm that deep astrophysical nulls (of the order of 0.001) can already be reliably measured (with $1 \sigma$ error bars down to a few 0.0001) when applying our statistical data analysis to on-sky near infrared interferometric data. In fact there is little that is specific to the PFN instrument in our approach. The statistical data reduction could in principle be applied to any 2-beam interferometer working around null with a fringe tracker. Since null and visibility measurements are equivalent, the statistical analysis may then prove also useful to conduct regular visibility interferometry at high precision.

\subsection{Laboratory results}

The broadband nulling results that we present in this section have been obtained at JPL's nulling lab in July 2009. When used at the laboratory, the telescope light feeding the instrument is replaced by a reflective injection bench that mimic the Hale telescope. Two different infrared light sources have been used for this experiment: 


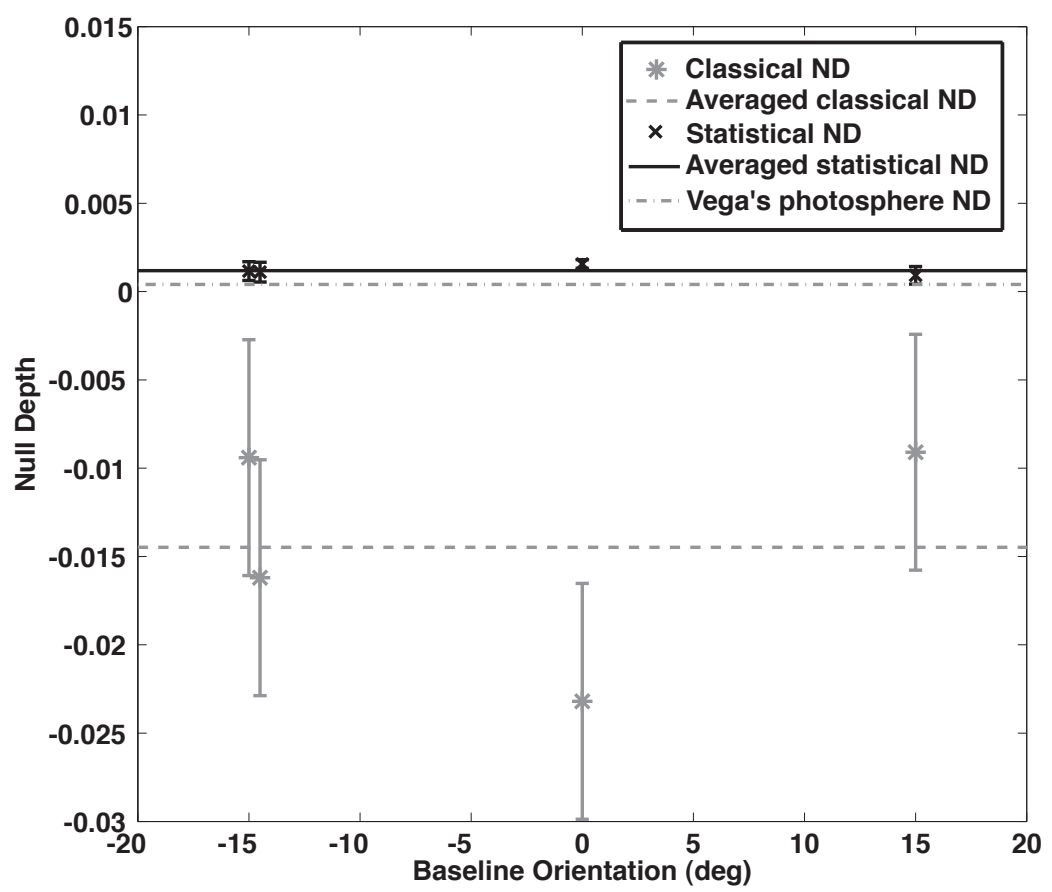

Figure 4. Astrophysical ND measured vs time on Vega with the PFN. Five individual sequences at different azimuths are shown, showing a mean null level of $0.0011 \pm 0.0005$. In grey, the same data points analyzed with a classical method show negative nulling values around $-1.5 \%$. It clearly shows the limitations of such a calibration method.

a classic Halogen lamp and a Fianium 2W supercontinuum white light source. The operational bandwidth of the instrument is set by the combination of the detector response with the $\mathrm{K}$ filter bandpass. The estimated bandpass of the instrument is $[2.05 ; 2.35] \mu \mathrm{m}$ which corresponds to a $14 \%$ bandwidth.

The result of the experiment is shown in Fig. 5. The best fitted histogram model correspond to a null depth of $1.0 \times 10^{-4}$ and is stable over the whole nulling sequence $(120 \mathrm{sec})$. The intensity mismatch during the measurement is $3.08 \%$ and the mean phase error corresponding to this best fit is $0.03 \mathrm{rad}$. Despite the huge power difference between the two sources used during the different nulling trials, no significant differences have been measured in terms of contrasts. This clearly shows that the contrast is not currently limited by the signal to noise ratio but by instrumental effects. This result illustrates the instrumental bias that we should expect from the PFN on-sky observations.

\section{CONCLUSIONS}

The theory of a new data reduction method for interferometric nulling (or visibility) observations has been presented in this paper. Based on the analysis of null distributions, this technique allows to retrieve high dynamic range astrophysical measurements, at contrast levels far exceeding the usual limits set by mean instrumental performance and fluctuations. The ultimate performance of this statistical data reduction depends on the specific design of the interferometric instrument and on the observing strategy. It is generally applicable to any interferometric set-up using a fringe tracking capability and any type of beam recombination (co-axial or multi-axial) into a single-mode waveguide. A similar statistical analysis may also be conducted in coronagraphic instruments in order to improve the measurement of stellar diameters. ${ }^{9}$

Applying our data reduction method to stellar observations obtained at K-band $(\simeq 2.2$ microns) with the first generation fiber nulling instrument installed at the Palomar $5 \mathrm{~m}$ (Hale) telescope, we demonstrated for the first time that: (i) contrast levels of the order of 0.001 can be obtained well within the diffraction limit of a large AO equipped aperture, (ii) deep and accurate nulling is no longer restricted to mid-infrared wavelengths but also accessible to the near infrared domain, providing substantial gains in resolution and sensitivity, (iii) null depth 


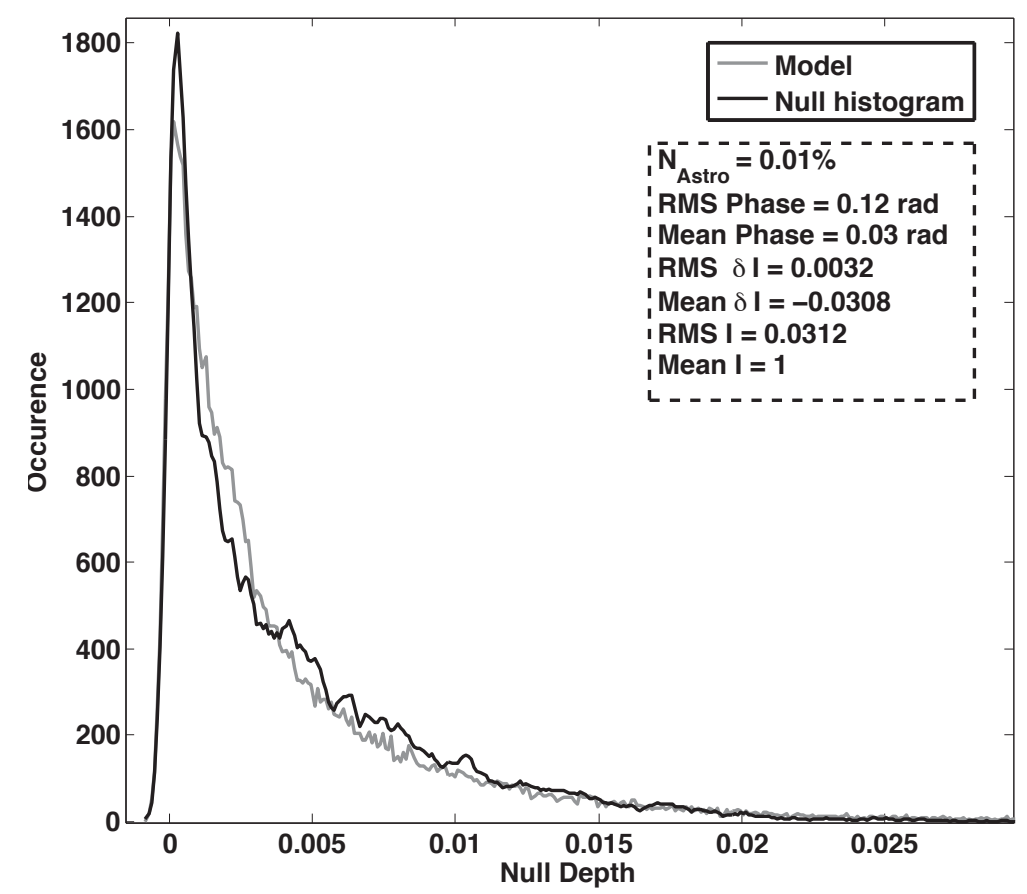

Figure 5. Comparison of the nulling histogram with the best fitted statistical model. The corresponding null depth is $10^{-4}$ with a mean intensity missmatch of $\mu_{\delta I}=3.08 \%$ and a mean phase error $\mu_{\Delta \phi}=0.03 \mathrm{rad}$.

accuracies of a couple of $10^{-4}$ level can be achieved without any observation of calibrator stars and corresponds to visibility accuracies of 0.0004 . Although this remains to be validated with an optimized instrument, (e.g. a second generation PFN), simulations suggest that this new analysis will enable direct detection of faint structures at the 0.0001 level within the near diffraction limit of large AO equipped ground based telescopes, i.e at angular separations ranging from $\simeq 20$ to 150 mas. Results obtained in the lab have indeed shown that the PFN instrumental bias is as low as 0.0001 when the data is processed using the statistical self calibration method. Implications for long baseline interferometry, both from the ground and from space, remain to be quantitatively explored. But since the statistical approach allows to detect astrophysical signals much below the mean contrast level and its rms fluctuations, we anticipate that the instrumental null stability requirements will be strongly relaxed. This implies that the constraints on intensity and phase fluctuations may be strongly reduced, a much attractive prospect for deep nulling interferometry from space in particular.

\section{ACKNOWLEDGMENTS}

This work was carried out at the Jet propulsion Laboratory, California Institute of Technology, under contract with the National Aeronautics and Space Administration. The research was supported by the Fond National de la Recherche scientifique de Belgique (FNRS), by the Fonds pour la formation à la Recherche dans l'Industrie et dans l'Agriculture de Belgique (FRIA) and by the Communauté Francaise de Belgique - Action de recherche concertée - Académie Wallonie - Europe and by the Center for Exoplanet Science.

\section{REFERENCES}

[1] Mayor, M. and Queloz, D., "A Jupiter-Mass Companion to a Solar-Type Star," Nature 378, 355-+ (Nov. 1995).

[2] Bracewell, R. N., "Detecting nonsolar planets by spinning infrared interferometer," Nature 274, 780-781 (Aug. 1978). 
[3] Colavita, M. M., Serabyn, E., Millan-Gabet, R., Koresko, C. D., Akeson, R. L., Booth, A. J., Mennesson, B. P., Ragland, S. D., Appleby, E. C., Berkey, B. C., Cooper, A., Crawford, S. L., Creech-Eakman, M. J., Dahl, W., Felizardo, C., Garcia-Gathright, J. I., Gathright, J. T., Herstein, J. S., Hovland, E. E., Hrynevych, M. A., Ligon, E. R., Medeiros, D. W., Moore, J. D., Morrison, D., Paine, C. G., Palmer, D. L., Panteleeva, T., Smith, B., Swain, M. R., Smythe, R. F., Summers, K. R., Tsubota, K., Tyau, C., Vasisht, G., Wetherell, E., Wizinowich, P. L., and Woillez, J. M., "Keck Interferometer Nuller Data Reduction and On-Sky Performance," PASP 121, 1120-1138 (Sept. 2009).

[4] Serabyn, E., "Nulling interferometry: symmetry requirements and experimental results," in [Proc. SPIE Vol. 4006, Interferometry in Optical Astronomy, Pierre J. Lena; Andreas Quirrenbach; Eds.], Lena, P. J. and Quirrenbach, A., eds., 328-339 (July 2000).

[5] Lay, O., "Systematic Errors in Nulling Interferometers," Appl. Opt. 43, 6100-6123 (2004).

[6] Mennesson, B., Haguenauer, P., Serabyn, E., and Liewer, K., "Deep broad-band infrared nulling using a single-mode fiber beam combiner and baseline rotation," in [Society of Photo-Optical Instrumentation Engineers (SPIE) Conference Series], Society of Photo-Optical Instrumentation Engineers (SPIE) Conference Series 6268 (July 2006).

[7] Haguenauer, P. and Serabyn, E., "Deep nulling of laser light with a single-mode-fiber beam combiner," Appl. Opt. 45, 2749-2754 (2006).

[8] Martin, S., Serabyn, E., Liewer, K., Loya, F., Mennesson, B., Hanot, C., and Mawet, D., "The development and applications of a ground-based fiber nulling coronagraph," in [Society of Photo-Optical Instrumentation Engineers (SPIE) Conference Series], Society of Photo-Optical Instrumentation Engineers (SPIE) Conference Series 7013 (July 2008).

[9] Riaud, P. and Hanot, C., "Combining coronagraphy with interferometry as a tool for measuring stellar diameters," Accepted in ApJ (2010).

[10] Cagigal, M. P. and Canales, V. F., "Residual phase variance in partial correction: application to the estimate of the light intensity statistics," J. Opt. Soc. Am. A 17(7), 1312-1318 (2000).

[11] Roddier, F., "Testing seeing quality," in [European Southern Observatory Astrophysics Symposia], J.P. Swings \& K. Kjaer, ed., European Southern Observatory Astrophysics Symposia 17, 255-261 (May 1983).

[12] Ten Brummelaar, T. and Bagnuolo, W. G., "CHARA beam combiner design," in [Proc. SPIE Vol. 2200, p. 140-151, Amplitude and Intensity Spatial Interferometry II, James B. Breckinridge; Ed.], 140-151 (June 1994).

[13] Coudé du Foresto, V., Perrin, G., Mariotti, J., Lacasse, M., and Traub, W., [The FLUOR/IOTA fiber stellar interferometer], 115-+, Integrated Optics for Astronomical Interferometry (1997).

[14] Millour, F., Tatulli, E., Chelli, A., Zins, G., Acke, B., and Malbet, F., "Data reduction for the AMBER instrument," in [New Frontiers in Stellar Interferometry. Edited by Wesley A., Traub, Proceedings of the SPIE, Volume 5491], 1222-1229 (Sept. 2004).

[15] Hinz, P. M., Angel, J. R. P., Hoffmann, W. F., McCarthy, D. W., McGuire, P. C., Cheselka, M., Hora, J. L., and Woolf, N. J., "First results of nulling interferometry with the Multiple-Mirror Telescope," in [Society of Photo-Optical Instrumentation Engineers (SPIE) Conference Series], R. D. Reasenberg, ed., Society of Photo-Optical Instrumentation Engineers (SPIE) Conference Series 3350, 439-447 (July 1998).

[16] Kervella, P., Coudé du Foresto, V., Segransan, D., and di Folco, E., "Optimal interferometric data acquisition and processing: towards $0.1 \%$ precision with the single-mode beam combiner VINCI," in [Society of Photo-Optical Instrumentation Engineers (SPIE) Conference Series], W. A. Traub, ed., Society of PhotoOptical Instrumentation Engineers (SPIE) Conference Series 5491, 741-+ (Oct. 2004).

[17] Perrin, G., Ridgway, S. T., Coudé du Foresto, V., Mennesson, B., Traub, W. A., and Lacasse, M. G., "Interferometric observations of the supergiant stars $\alpha$ Orionis and $\alpha$ Herculis with FLUOR at IOTA," $A \& A$ 418, 675-685 (May 2004).

[18] Aufdenberg, J. P., Mérand, A., Coudé du Foresto, V., Absil, O., Di Folco, E., Kervella, P., Ridgway, S. T., Berger, D. H., ten Brummelaar, T. A., McAlister, H. A., Sturmann, J., Sturmann, L., and Turner, N. H., "First Results from the CHARA Array. VII. Long-Baseline Interferometric Measurements of Vega Consistent with a Pole-On, Rapidly Rotating Star," ApJ 645, 664-675 (July 2006). 